\title{
Cost-effectiveness analysis of lamivudine, telbivudine, and entecavir in treatment of chronic hepatitis $B$ with adefovir dipivoxil resistance
}

This article was published in the following Dove Press journal:

Drug Design, Development and Therapy

2 June 2015

Number of times this article has been viewed

\author{
Guiliang Wang ${ }^{1,2}$ \\ Yan Liu $^{2}$ \\ Ping Qiu' \\ Shu-Feng Zhou ${ }^{3,4}$ \\ Linfang $X u^{\prime}$ \\ Ping Wen' \\ Jianbo Wen' \\ Xianzhong $\mathrm{Xiao}^{5}$
}

'Department of Digestive Internal Medicine, Gannan Medical University Pingxiang Hospital, Pingxiang, People's Republic of China; ${ }^{2}$ Department of Digestive Internal Medicine, 307 Hospital of PLA, Beijing, People's Republic of China; ${ }^{3}$ Department of Pharmaceutical Sciences, College of Pharmacy, University of South Florida, Tampa, FL, USA; ${ }^{4}$ Guizhou Provincial Key Laboratory for Regenerative Medicine, Stem Cell and Tissue Engineering Research Center and Sino-US Joint Laboratory for Medical Sciences, Guiyang Medical University, Guiyang, People's Republic of China; ${ }^{5}$ Laboratory of Shock, Department of Pathophysiology, Xiangya School of Medicine, Central South University, Changsha, People's Republic of China

Correspondence: Xianzhong Xiao Department of Pathophysiology, Xiangya School of Medicine, Central South University, I 10 Xiangya Road, Changsha 4I0008, Hunan, People's Republic of China

Tel +867312355019

Fax +867312355019

Email csxianzhongxiaoxy@।26.com
Abstract: The purpose of this study was to analyze the cost-effectiveness of lamivudine (LMV), telbivudine (LdT), and entecavir (ETV) in treatment of chronic hepatitis B with adefovir dipivoxil (ADV) resistance. Two hundred and fifty-two patients were recruited and screened for resistance to ADV and randomly assigned into three groups: $\mathrm{LMV}+\mathrm{ADV}, \mathrm{LdT}+\mathrm{ADV}$, and ETV + ADV. The ratio of biochemical response, virological response, seroconversion of hepatitis Be antigen (HBeAg)/hepatitis Be antibody (HBeAb), viral breakthrough, and the cost and effectiveness of treatments were analyzed. A comparison of the results of the ratio of biochemical response, virological response and seroconversion of $\mathrm{HBe} \mathrm{Ag} / \mathrm{HBe} \mathrm{Ab}$, showed no statistical difference between the three groups, with the economic cost of LMV + ADV the lowest, LdT + ADV the middle, and ETV + ADV the highest. The side effects of the three plans are all rare and tolerable. LMV + $\mathrm{ADV}$ is the optimal rescue strategy, and LdT + ADV the alternative selection in the economically less developed regions, while ETV + ADV was used in the economically developed regions.

Keywords: chronic hepatitis B, adefovir dipivoxil, lamivudine, telbivudine, entecavir, resistance

\section{Introduction}

Chronic infection with the chronic hepatitis B virus (HBV) is a leading cause of liverrelated morbidity and mortality. ${ }^{1,2}$ It is estimated that approximately two billion people are infected with HBV in the world, which results in approximately 500,000 deaths every year, mainly due to its complications including cirrhosis and hepatocellular carcinoma (HCC). ${ }^{3,4}$ Evidence-based medicine has demonstrated that effective antiviral treatment of chronic hepatitis $\mathrm{B}(\mathrm{CHB})$ can reduce the risk of long-term complications and improve patient survival. ${ }^{5,6}$ Current nucleos(t)ide analogs (NUCs) approved by the US Food and Drug Administration (FDA) for CHB patients include lamivudine (LMV), adefovir dipivoxil (ADV), entecavir (ETV), tenofovir disoproxil fumarate (TDF), and telbivudine (LdT). ${ }^{7,8}$ Though ETV and TDF have been recommended as the first-line options for treatment of naïve CHB patients, they are not in widespread used in countries with limited health resources due to the high daily cost or difficulty of availablity, ${ }^{9,10}$ and therefore LMV and ADV are still widely used in the world, especially in the economically less developed regions due to their low cost and easy availablity. ${ }^{11,12}$ ADV was recommended by certain scholars because it has less drug resistance ratio than LMV, while LMV was the earliest available pharmacon and was still used frequently in many economically less developed countries. The ratio of therapy with ADV for 1-3 years associatied with mutations in the polymerase (particularly in N236T or N181T) is about $0 \%, 1.6 \%$, and $3.1 \%$, respectively. ${ }^{13}$ If the HBV develops a mutant, ADV is 
recommended to be used continuously and combinative with a second drug without cross-resistance such as LMV, LdT, and ETV. This is because the HBV reproduce quickly and it is making it very difficult for sequential monotherapy to prevent the virus reproduction, which results in significantly lower probability of virologic response and significantly higher risk of virologic breakthrough in the "switch" group than in the "add-on" group. ${ }^{14}$ Because the cost of the LMV, LdT, or ETV is greatly different from each other, we aim to compare the cost-effectiveness of the three pharmacons for treatment of chronic hepatitis B with adefovir dipivoxil resistance.

\section{Materials and methods \\ Patients}

This is a prospective double-blind study, and a total of 252 patients diagnosed with $\mathrm{CHB}$ who were admitted to our hospital between January 2006 and August 2014 were recruited and screened for resistance to ADV. CHB patients who met the following criteria were included in the study: serum chronic hepatitis B e antigen positive and serum HBV DNA level $\geq 500$ copies $/ \mathrm{mL}$. ADV resistance was defined on the basis of genetic testing (RtN236T or RtN181T) or presence of virological breakthrough, ${ }^{15}$ which we defined as a documented rise in serum HBV DNA by $\geq 1 \log _{10} \mathrm{IU} / \mathrm{mL}$ above the nadir, or to a detectable level ( $\geq 500$ copies $/ \mathrm{mL}$ ) after achieving virological response while continuing ADV therapy. Exclusion criteria were: previous treatment for chronic hepatitis B with LMV, LdT, or ETV; coinfection with hepatitis $\mathrm{C}$ or human immunodeficiency virus; other forms of liver disease; breast-feeding, pregnancy or inadequate contraceptive measures; coexisting serious medical disease; and evidence of hepatic decompensation or HCC. Demographic data, factors of sex, age, alanine aminotransferase (ALT), and HBV DNA were not statistically different between the three subgroups (Table 1).

Table I Baseline characteristics of the study population

\begin{tabular}{|c|c|c|c|c|}
\hline Group & $\begin{array}{ll}\text { n } & \begin{array}{l}\text { Age } \\
\text { (mean } \pm \text { SD }\end{array} \\
& \text { (years) }\end{array}$ & $\begin{array}{l}\text { Males/ } \\
\text { females }\end{array}$ & $\begin{array}{l}\text { ALT } \\
\text { (IU/L) }\end{array}$ & $\begin{array}{l}\text { HBV DNA } \\
\left(\log _{10} \text { copies } / \mathrm{mL}\right)\end{array}$ \\
\hline LMV + ADV & $884 \mathrm{I} \pm \mathrm{II}$ & $45 / 43$ & $137.9 \pm 37.1$ & $4.98 \pm 1.34$ \\
\hline LdT + ADV & $8438 \pm 13^{*}$ & $43 / 4 I^{*}$ & $146.7 \pm 30.9 *$ & $5.14 \pm 2.13 *$ \\
\hline $\mathrm{ETV}+\mathrm{ADV}$ & $8040 \pm 12^{* *}$ & $42 / 38 * *$ & $138.9 \pm 32.4 * *$ & $5.02 \pm 1.36 * *$ \\
\hline$P$-value & 0.644 & 0.718 & 0.857 & 0.784 \\
\hline
\end{tabular}

Notes: *, vs LMV + ADV group, $P>0.05$; **, vs LdT + ADV group, $P>0.05$.

Abbreviations: ALT, alanine aminotransferase; $H B V$, hepatitis $B$ virus; $L M V$, lamivudine; ADV, adefovir dipivoxil; LdT, telbivudine; ETV, entecavir.

\section{Treatment of patients}

The patients were randomly divided into three groups: $\mathrm{LMV}+\operatorname{ADV}(\mathrm{n}=88), \mathrm{LdT}+\operatorname{ADV}(\mathrm{n}=84), \mathrm{ETV}+\mathrm{ADV}$ $(\mathrm{n}=80)$. Drug usage ${ }^{16,17}$ : LMV (GlaxoSmithKline Pharmaceutical Company Limited, London, UK) $100 \mathrm{mg}$ oral administration once a day; LdT (Beijing Novartis Pharma Ltd, Beijing, People's Republic of China) 600 mg oral administration once a day; ADV (GlaxoSmithKline Pharmaceutical Company Limited) $10 \mathrm{mg}$ oral administration once a day; ETV (Bristol-Myers Squibb Pharmaceutical Company Limited, New York, NY, USA) $0.5 \mathrm{mg}$ oral administration once a day. This study was approved by the institutional review board at Gannan Medical University and was conducted in accordance with the 1975 Declaration of Helsinki.

\section{Biochemical and serologic responses}

A peripheral blood sample was taken from the patients in the morning, after fasting for at least 8 hours. Hepatitis B surface antigen (HBsAg), anti-HBsAg, hepatitis Be antigen (HBeAg), anti-HBe, anti-hepatitis B core (HBc) (total), and anti-hepatitis $\mathrm{B}$ core (HBc IgM were identified by using the third-generation microparticle enzyme immunoassays (Abbott Laboratories, Chicago, IL, USA). Serum alanine aminotransferase (ALT), aspartate aminotransferase levels, creatinine, and creatine kinase were measured with an automatic biochemistry analyser (Olympus AU5400, Tokyo, Japan) according to standard laboratory procedures. The biochemical response was defined as normalization of ALT levels.

\section{Virological analysis}

HBV DNA was investigated by real-time polymerase chain reaction (RT-PCR) (COBAS TaqMan 48 analyzer; Roche DiagnosticGmbH, Shanghai, People's Republic of China) according to manufacturer's instructions. Virological response was defined as a decrease in serum HBV DNA to undetectable levels by PCR assays ( $<500$ copies/mL). HBeAg response was defined as seroconversion of HBeAg. Virological breakthrough was defined as an increase in serum HBV DNA by $1 \log _{10} \mathrm{IU} / \mathrm{mL}$ above nadir, or to detectable level ( $\geq 500$ copies $/ \mathrm{mL}$ ) after achieving virological response during retreatment.

\section{Genotypic analysis}

HBV mutations were analyzed by direct sequencing. Extracted from serum samples, the HBV DNA polymerase gene region was amplified by nested-PCR using specific primers. We employed the primers and PCR program of Osiowy et al. ${ }^{18}$ Briefly, the primers, forward primer: spr $1 \mathrm{~F}$ (5'-GTTCAGGAACAGTAAGCCC-3') and the reverse 
primer: spr1R (5'- GAAAGGCCTTGTAAGTTGGCG-3'), were used in the first round PCR. The inner primers: sense, spr2F (5'-GGTGGACTTCTCTCAATTTTCTAGG-3') and antisense, spr2R (5'-ACTTTCCAATCAATAGGCC-3') were used for nested PCR. The following PCR thermalcycling program was performed: 35 cycles at $94^{\circ} \mathrm{C}$ for 30 seconds, $56^{\circ} \mathrm{C}$ for 30 seconds (first round) or $50^{\circ} \mathrm{C}$ for 30 seconds (second round), and $72^{\circ} \mathrm{C}$ for 40 seconds. The intended fragments were amplified using $2 \times$ PCR master mix solution (Tiangen Biotech Co., Ltd, Beijing, People's Republic of China) with $5 \mu \mathrm{L}$ of DNA extract and $2 \mu \mathrm{L}$ of the first round PCR product. After amplification of the polymerase gene, the amplicons (730 bp) were visualized by agarose gel electrophoresis and gel purified using High Pure PCR Product purification kit (Roche Diagnostic $\mathrm{GmbH}$ ). The purified PCR products were bidirectionally sequenced commercially (SEQLAB, Gottingen, Germany) using inner primers.

\section{Cost calculation}

A cost calculation was performed by means of identification and subsequent quantification of resources used, and assigning unitary cost to each. The costs are presented in dollars, for the year 2008. Unitary cost of LMV, LdT, ADV, and ETV was US\$1.8, 2.2, 2.7, and 4.9 respectively.

\section{Cost-effectiveness ratios (CER) and incremental cost-effectiveness ratio (ICER)}

Cost effectiveness ratio (CER), standing for the cost for each unit of effectiveness produced by each therapeutic plan, was computed by dividing the difference in the mean costs of the three therapies (referred to as costs) and was assessed by the following formulas: $\mathrm{CER}_{\mathrm{LMV}+\mathrm{ADV}}=\mathrm{Cost}_{\mathrm{LMV}+\mathrm{ADV}} /$ Effectiveness $_{\mathrm{LMV}+\mathrm{ADV}}, \mathrm{CER}_{\mathrm{LdT}+\mathrm{ADV}}=\operatorname{Cost}_{\mathrm{LdT}+\mathrm{ADV}} /$ Effectiveness $_{\mathrm{LdT}+}$ ${ }_{\mathrm{ADV}}, \mathrm{CER}_{\mathrm{ETV}+\mathrm{ADV}}=\mathrm{Cost}_{\mathrm{ETV}+\mathrm{ADV}} /$ Effectiveness $_{\mathrm{ETV}+\mathrm{ADV}}$

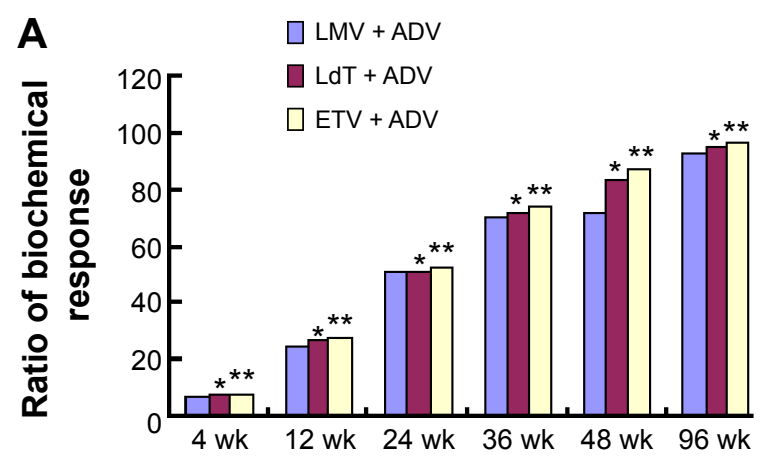

With reference to LMV, the ICER, defined as the additional cost incurred to achieve an extra unit of effectiveness, was calculated by applying the following formula: ICER $_{\mathrm{LdT}}$ $+\mathrm{ADV}$ vs $\mathrm{LMV}+\mathrm{ADV}=\left(\operatorname{Cost}_{\mathrm{LdT}+\mathrm{ADV}}-\operatorname{Cost}_{\mathrm{LMV}+\mathrm{ADV}}\right) /($ Effective ness $_{\mathrm{LdT}+\mathrm{ADV}}-$ Effectiveness $\left._{\mathrm{LMV}+\mathrm{ADV}}\right), \mathrm{ICER}_{\mathrm{ETV}}+\mathrm{ADV}$ vs LMV + $\mathrm{ADV}=\left(\operatorname{Cost}_{\mathrm{ETV}}+\mathrm{ADV}_{\mathrm{ADV}}-\operatorname{Cost}_{\mathrm{LMV}}+\mathrm{ADV}_{\mathrm{ADV}}\right) /\left(\right.$ Effectiveness $_{\mathrm{ETV}}+\mathrm{ADV}$ - Effectiveness $\left._{\text {LMV }+ \text { ADV }}\right)$.

\section{Sensitivity analyses}

The uncertainties were minimized with sensitivity analyses that were performed using univariate analysis, varying the parameters of cost and effectiveness by either increasing or decreasing the value. To evaluate the robustness of the method and reduce uncertainties, the following types of analyses were performed: varying $10 \%$ discount on the prices for each drug; increasing the dosage of LMV from 100 to $150 \mathrm{mg} /$ day; and adjusting the ratios of LMV arm response rate in $\mathrm{LMV}+\mathrm{ADV}$ at 96 weeks with the intention of achieving the "threshold values".

\section{Statistical analysis}

Statistical testing was performed by using SPSS version 12 (SPSS Inc., Chicago, IL, USA). The data are reported as median (range). Data are expressed as mean \pm SEM of the indicated number of separate experiments. Statistical comparison between experimental group and control was performed by using one-way ANOVA analysis and unpaired two-tailed Student's $t$-tests (for measurement data) or Chi-square test (for percentage). $P<0.05$ was considered significant.

\section{Results}

\section{Biochemical response and seroconversion of $\mathrm{HBeAg} /$ hepatitis Be antibody ( $\mathrm{HBeAb}$ )}

As shown in Figure 1A, at week 4, 12, 24, 36, 48, and 96 , the ratio of patients with biochemical response was

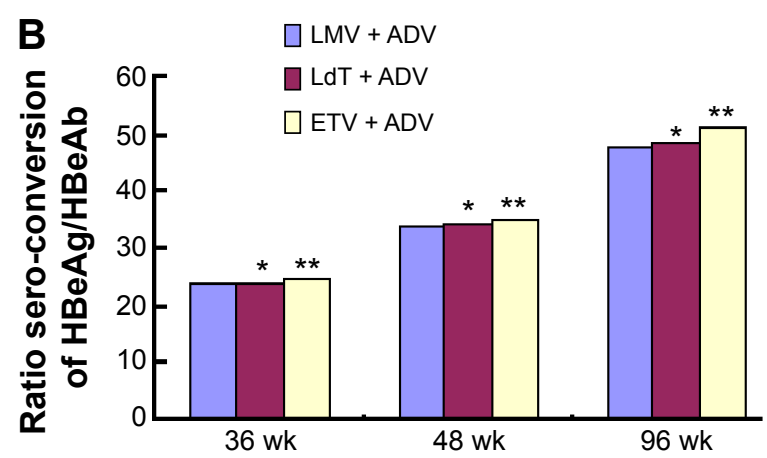

Figure I Ratio of biochemical response and seroconversion of $\mathrm{HBeAg} / \mathrm{HBeAb}$ of the three groups.

Notes: (A) Ratio of biochemical response; (B) ratio of seroconversion of HBeAg/HBeAb. *, vs LMV + ADV group, $P>0.05 ; * *$, vs LdT + ADV group, P>0.05. Abbreviations: LMV, lamivudine; ADV, adefovir dipivoxil; LdT, telbivudine; ETV, entecavir; wk, week. 
$6.8 \%, 25 \%, 51.1 \%, 70.5 \%, 72 \%$, and $93.1 \%$ in LMV + ADV group respectively; $7.1 \%, 27.4 \%, 51.2 \%, 72.6 \%$, $83.3 \%$, and $95.2 \%$ respectively in the LdT + ADV group; $7.5 \%, 27.5 \%, 52.5 \%, 75 \%, 87.5 \%$, and $97.5 \%$ respectively in the ETV + ADV group. There were no differences in virological response between the three groups. As shown in Figure 1B, at weeks 36, 48, and 96 of treatment, ratio of seroconversion of $\mathrm{HBeAg} / \mathrm{HBeAb}$ was $23.7 \%, 34.1 \%$, and $47.7 \%$ respectively in the LMV + ADV group; $23.8 \%$, $34.5 \%$, and $48.8 \%$ respectively in the LdT + ADV group; and $25.0 \%, 35.0 \%$, and $51.3 \%$ respectively in the ETV + ADV group. There were no differences between the ratios of seroconversion of $\mathrm{HBeAg} / \mathrm{HBe} \mathrm{Ab}$ in the three groups.

\section{Virological response}

As shown in Figure 2A, at weeks 4, 12, 24, 36, 48, and 96, HBV DNA levels decreased to $4.18 \pm 1.13,3.62 \pm 0.38$, $2.76 \pm 0.36,2.03 \pm 0.23,1.76 \pm 0.12$, and $0.31 \pm 0.42$ respectively in the LMV + ADV group; to 4.13 $\pm 1.03,3.26 \pm 0.62$, $2.63 \pm 0.28,1.94 \pm 0.43,1.65 \pm 0.16$, and $0.28 \pm 0.36$ respectively in the $\mathrm{LdT}+\mathrm{ADV}$ group; and to $3.98 \pm 0.92,3.14 \pm 0.70$, $2.43 \pm 0.09,1.89 \pm 0.34,1.55 \pm 0.12$, and $0.26 \pm 0.38$ respectively in the ETV + ADV group. As shown in Figure 2B, at week $4,12,24,36,48$, and 96 , the ratio of patients with virological responses was $6.8 \%, 26.1 \%, 65.9 \%, 76.1 \%, 84.1 \%$, and $90.9 \%$ in LMV + ADV group respectively; 7.1\%, 26.2\%, $66.7 \%, 77.4 \%, 85.7 \%$, and $92.9 \%$ respectively in the LdT + ADV group; and 7.5\%, 27.5\%, 70\%, 78.7\%, 87.5\%, and $93.8 \%$ respectively in the ETV + ADV group. There were no differences between the virological response between the three groups.

\section{Viral breakthrough and resistance}

At week 96 of treatment, three patients (3.4\%) developed viral breakthrough and genotypic mutation, including two RtM204V and one RtL180M, in the LMV + ADV group; two patients $(2.2 \%)$ developed viral breakthrough and genotypic mutation, including one RtA181V and one RtN236T, in the LdT + ADV group; and two patients (2.5\%) developed breakthrough and genotypic mutation, including one RtN236T and one RtL180M + RtT184G, in the ETV + ADV group. There were no statistical differences between the ratios of viral breakthrough in the three groups (Table 2).

\section{Cost-effectiveness and incremental cost- effectiveness ratio analysis of the three groups}

At week 96 of treatment, in the LMV + ADV, LdT + ADV, and ETV + ADV group, the cost was US\$3,024, US\$3,292.8 and US\$5,107.2 respectively, the ratio of negative conversion of HBV DNA was 90.9, 92.9, and 93.8 respectively, the costeffectiveness ratio of negative conversion of HBV DNA was $33.3,35.4$, and 544 respectively; Ratio of seroconversion of $\mathrm{HBe} \mathrm{Ag} / \mathrm{HBeAb}$ was 47.7, 48.8, and 51.3 respectively, costeffectiveness ratio of seroconversion of $\mathrm{HBeAg} / \mathrm{HBeAb}$ was $63.4,67.5$, and 99.5 respectively; Ratio of non-genotypic mutation ratio was $96.6 \%, 97.6 \%$, and $97.5 \%$ respectively, cost-effectiveness ratio of non-genotypic mutation was $31.3,33.7$, and 52.4 respectively. In reference to $\mathrm{LMV}+\mathrm{ADV}$, incremental cost-effectiveness ratio of negative conversion of HBV DNA of LdT + ADV and ETV + ADV group was 134.4 and 718.3 respectively, incremental cost-effectiveness ratio of seroconversion of $\mathrm{HBe} \mathrm{Ag} / \mathrm{HBeAb}$ was 244.4 and
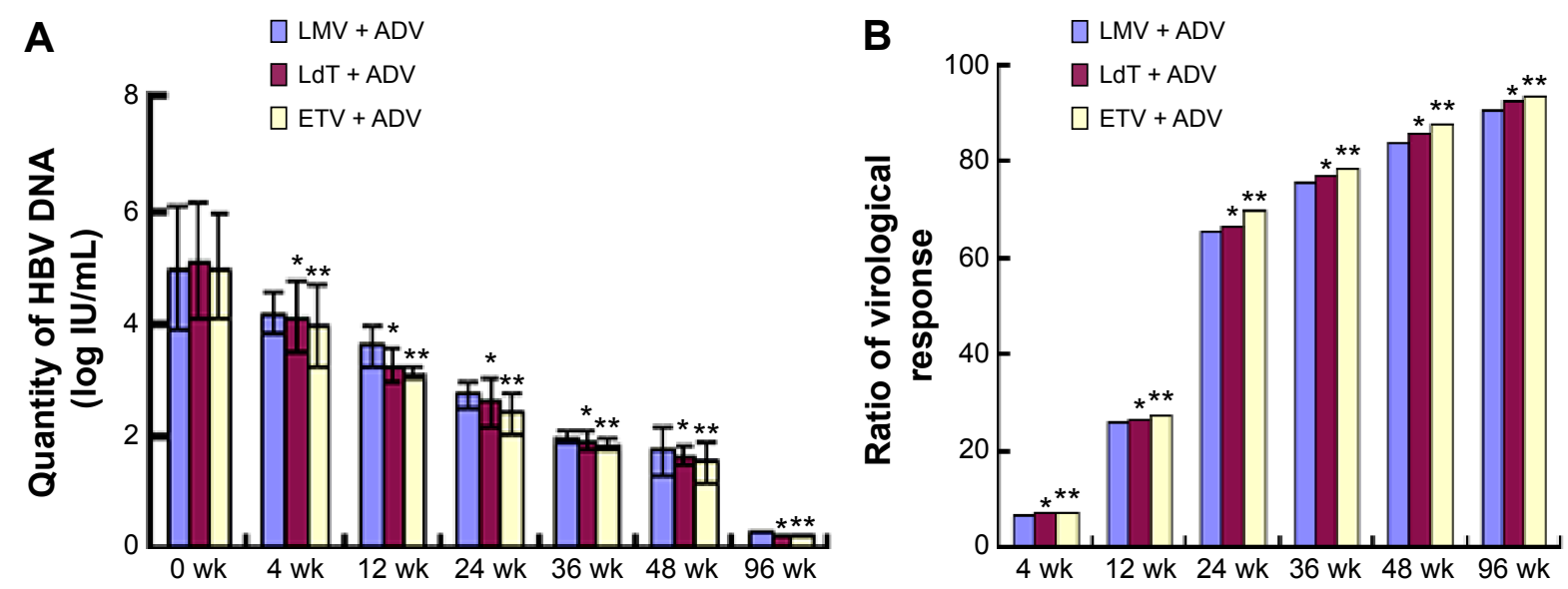

Figure 2 Virological response of the three groups.

Notes: (A) Quantity of HBV DNA; (B) ratio of virological responses. *, vs LMV + ADV group, P>0.05; **, vs LdT + ADV group, P>0.05.

Abbreviations: LMV, lamivudine; ADV, adefovir dipivoxil; LdT, telbivudine; ETV, entecavir; HBV, hepatitis B virus; wk, week. 
Table 2 Viral breakthrough and genotypic mutation of the three groups

\begin{tabular}{lllll}
\hline Group & $\mathbf{n}$ & $\begin{array}{l}\text { Genotypic } \\
\text { mutation }(\mathbf{n}, \%)\end{array}$ & $\begin{array}{l}\text { Nongenotypic } \\
\text { mutation (n, \%) }\end{array}$ & $\begin{array}{l}\text { Mutation } \\
\text { genotype (n) }\end{array}$ \\
\hline LMV + ADV & 88 & $3(3.4)$ & $85(96.6)$ & RtM204V (2), RtLI80M (I) \\
LdT + ADV & 84 & $2(2.4)^{*}$ & $82(97.6)^{*}$ & RtAI8IV (I), RtN236T (I) \\
ETV + ADV & 80 & $2(2.5)^{* * * *}$ & $78(97.5)^{* * * *}$ & $\begin{array}{l}\text { RtN236T (I), } \\
\text { RtLI80M + RtTI84G (I) }\end{array}$ \\
P-value & & & 0.631 & \\
\hline
\end{tabular}

Notes: *, vs LMV + ADV group, $P>0.05 ;$ **, vs LdT + ADV group, $P>0.05$.

Abbreviations: LMV, lamivudine; ADV, adefovir dipivoxil; LdT, telbivudine; ETV, entecavir.

578.7 respectively, incremental cost-effectiveness ratio of nongenotypic mutation was 268.8 and 2,314.7 respectively. These results showed that LMV was the least economically costwise, LdT the middle, and ETV the most economically costwise (Table 3).

\section{Sensitivity analyses}

In the three groups of patients, varying the discount rate to $10 \%, \mathrm{LMV}+\mathrm{ADV}$ was more cost-effective than LdT + ADV and ETV + ADV. If LMV dose increases to $150 \mathrm{mg}$ or the ratio of virological response increases to $91.9 \%$ at 96 weeks, there is a favorable impact on the average cost-effectiveness analysis for LMV + ADV. The sensitivity analysis demonstrates the robustness of the results obtained with the base case scenario with the three variables significantly impacting the results (Table 4).

\section{Side effects}

All the patients tolerated treatment well, and no patient discontinued the therapy. At week 36 of treatment, ratio of side effects happening in the LMV + ADV group was $1.1 \%$, with one patient demonstrating blood urea nitrogen (BUN) elevation $(14.2 \mathrm{mmol} / \mathrm{L})$, ratio of side effects in
LdT + ADV group was $1.2 \%$, with one patient demonstrating BUN elevation $(14.7 \mathrm{mmol} / \mathrm{L})$ and one patient with creatine kinase elevation $(138.6 \mu \mathrm{mol} / \mathrm{L})$, ratio of side effects happening in the ETV + ADV group was $1.3 \%$, with one patient demonstrating BUN elevation $(14.6 \mathrm{mmol} / \mathrm{L})$. At week 96 of treatment, ratio of side effects happening in the LMV + ADV group was $4.5 \%$, with two patients having blood urea BUN elevation (mean $13.9 \mathrm{mmol} / \mathrm{L}$ ), one patient having diarrhea, and one patient having nausea; ratio of side effects in LdT + ADV group was $7.1 \%$, with two patients having BUN elevation (mean $14.5 \mathrm{mmol} / \mathrm{L}$ ), one patient having creatine kinase elevation $(142.3 \mu \mathrm{mol} / \mathrm{L})$; ratio of side effects in ETV + ADV group was $5.0 \%$, with two patients having BUN elevation (mean $13.9 \mathrm{mmol} / \mathrm{L}$ ), one patient having headache, and one patient having dizziness. The results showed there were statistical differences between the ratios of side effects between LdT + ADV group and LMV + ADV or ETV + ADV group. No statistical differences were observed between the ratios of LMV + ADV group and ETV + ADV group (Table 5). Acute renal failure or myopathy was not observed in any patient during the rescue therapy. The patients suffering side effects recovered after undergoing symptomatic treatments.

Table 3 Cost-effectiveness and incremental cost-effectiveness analysis of the three groups

\begin{tabular}{|c|c|c|c|c|}
\hline Group & LMV + ADV & LdT + ADV & ETV + ADV & $P$-value \\
\hline Cost (US\$) & 3,024 & $3,292.8$ & $5,107.2$ & \\
\hline Ratio of negative conversion of HBV DNA (\%) & 90.9 & $92.9 *$ & $93.8^{* * * *}$ & 0.418 \\
\hline Cost-effectiveness ratio of negative conversion of HBV DNA & 33.3 & 35.4 & 544 & \\
\hline Incremental cost-effectiveness ratio of negative conversion of HBV DNA & - & 134.4 & 718.3 & \\
\hline Ratio of seroconversion of $\mathrm{HBeAg} / \mathrm{HBeAb}(\%)$ & 47.7 & $48.8^{*}$ & $51.3^{* * * *}$ & 0.852 \\
\hline Cost-effectiveness ratio of seroconversion of $\mathrm{HBeAg} / \mathrm{HBeAb}$ & 63.4 & 67.5 & 99.5 & \\
\hline Incremental cost-effectiveness ratio of seroconversion of $\mathrm{HBeAg} / \mathrm{HBeAb}$ & - & 244.4 & 578.7 & \\
\hline Ratio of non-genotypic mutation (\%) & 96.6 & 97.6 & $97.5^{* * * *}$ & 0.742 \\
\hline Cost-effectiveness ratio of non-genotypic mutation & 31.3 & 33.7 & 52.4 & \\
\hline Incremental cost-effectiveness ratio of non-genotypic mutation & - & 268.8 & $2,314.7$ & \\
\hline
\end{tabular}

Notes: *, vs LMV + ADV group, $P>0.05$; **, vs LdT + ADV group, $P>0.05$.

Abbreviations: HBV, hepatitis B virus; LMV, lamivudine; ADV, adefovir dipivoxil; LdT, telbivudine; ETV, entecavir. 
Table 4 Sensitivity analysis

\begin{tabular}{|c|c|c|c|}
\hline & CER of LMV + ADV & ICER of LdT + ADV & ICER of ETV + ADV \\
\hline $10 \%$ discount on the prices & 29.97 & 120.06 & 646.47 \\
\hline Varying the discount rate to $10 \%$ & 33.3 & 134.4 & 718.3 \\
\hline Lamivudine dose is $150 \mathrm{mg}$ & 39.9 & -167.6 & 510.1 \\
\hline Ratio of virological response in LMV + ADV at 96 weeks is $91.9 \%$ & 32.9 & 268.6 & $1,096.4$ \\
\hline
\end{tabular}

Abbreviations: CER, cost-effectiveness ratio; LMV, lamivudine; ADV, adefovir dipivoxil; ICER, incremental cost-effectiveness ratio; LdT, telbivudine; ETV, entecavir.

\section{Discussion}

Persistent HBV infection is an important risk factor for the development of hepatic cirrhosis or for the occurrence of HCC. ${ }^{19}$ Oral nucleos(t)ide analogs have been widely used for more than 10 years, and the sustained suppression of serum HBV DNA to very low or undetectable levels has been associated with the prevention of liver disease progression and inhibition of the development of long-term complications. ${ }^{20}$ However, drug resistance has presented a serious challenge to CHB treatment. Insufficient antiviral efficacy caused by drug resistance has resulted in attenuated viral suppression, which can lead to significant clinical deterioration. LMV, LdT, and ETV can all be used as rescue strategies for HBeAg-positive chronic hepatitis $B$ patients with resistance to ADV because all of them have no resistant mutations of ADV in the HBV polymerase and can quickly suppress the replication of HBV. ${ }^{21-25}$ In the present study, in the LMV + ADV, LdT + ADV, and ETV + ADV groups, a sustainably increasing proportion of patients achieved undetectable HBV DNA levels from 4 to 96 weeks, and a sustainably increasing proportion of patients achieved seroconversion of $\mathrm{HBe} \mathrm{Ag} / \mathrm{HBeAb}$ from 36 to 96 weeks, which indicates that all the three plans have good prognosis, because the negative conversion of $\mathrm{HBV}$ is a predictor for the decreasing impairment of liver function, and hepatitis B e antigen seroconversion is a predictor for lower ratios of cirrhosis and slower disease progression. At week 96 of treatment, the ratio of patients experiencing viral breakthrough was $3.4 \%$, with three resistance mutation (two RtM204V and one RtL180M) in LAM + ADV group, the ratio of viral breakthrough was $2.4 \%$ in $\mathrm{LdT}+\mathrm{ADV}$ group, with two resistance mutation (one RtA181V and one

Table 5 Side effects of the three groups

\begin{tabular}{llll}
\hline Group & $\mathbf{n}$ & $\begin{array}{l}\text { Ratio of side } \\
\text { effects at 36 weeks }\end{array}$ & $\begin{array}{l}\text { Ratio of side } \\
\text { effects at } \mathbf{9 6} \text { weeks }\end{array}$ \\
\hline LMV + ADV & 88 & I (I.I\%) & $4(4.5 \%)^{* *}$ \\
LdT + ADV & 84 & $2(1.2 \%)^{*}$ & $6(7.1 \%)$ \\
ETV + ADV & 80 & I (I.3\%)* & $4(5.0 \%)^{* * * *}$ \\
\hline
\end{tabular}

Notes: *, vs LMV + ADV group, $P>0.05$; **, vs LdT + ADV group, $P<0.05$.

Abbreviations: LMV, lamivudine; ADV, adefovir dipivoxil; LdT, telbivudine; ETV, entecavir.
RtN236T), and the ratio of viral breakthrough was $2.5 \%$ in ETV + ADV group, with two resistance mutations (one RtN236T and one RtL180M + RtT184G), which showed that the ratios of viral breakthrough of the three plans are rare and can be controlled. Cost-effectiveness, incremental costeffectiveness ratio analysis, and sensitivity analyses prove that the cost, ratio and cost-effectiveness ratio of negative conversion of $\mathrm{HBV}$ DNA, seroconversion of $\mathrm{HBe} \mathrm{Ag} / \mathrm{HBe} \mathrm{Ab}$, and nongenotypic mutation of LMV + ADV were the least, the LdT + ADV the middle, and the ETV + ADV the most. At week 96 of treatment, ratio of side effects happening in the LMV + ADV, LdT + ADV and ETV + ADV group was $4.5 \%, 7.1 \%$, and $5.0 \%$ respectively. The main side effects of the four pharmacons are: for LMV, gastrointestinal symptom such as diarrhea and nausea; for ADV, impairment of the renal function; for LdT, creatine kinase elevation; and for ETV, gastrointestinal symptom (such as diarrhea and nausea) and nervous symptom (such as headache and dizziness). The cumulative side effects of combinative pharmacons are not evident.

LMV, a pharmacon of low cost, rare side effects, and high effect in inhibiting HBV replication and promoting $\mathrm{HBeAg}$ seroconversion, has been proven to be able to achieve regression of fibrosis/cirrhosis and, improve the histology and disease progression in advanced liver fibrosis/cirrhosis with chronic hepatitis B patients, ${ }^{26}$ so it is still a fairly good rescue pharmacon for adefovir dipivoxil resistance in the economically less developed regions, since rescue therapy can be carried out by using ETV or TDF, even if multidrug resistance to LMV and ADV happens. ${ }^{27,28}$ As another pharmacon of high effect in inhibiting HBV replication and promoting $\mathrm{HBeAg}$ seroconversion with less drug resistance but for a higher cost and a side effect of creatine kinase elevation, ${ }^{29} \mathrm{LdT}$ is an alternative rescue pharmacon for adefovir dipivoxil resistance in economically less developed regions. Because ETV has been proven to be able to significantly decrease the rates of compensated cirrhosis, decompensated cirrhosis, and hepatocellular carcinoma events with the least drug resistance among these four pharmacons, ${ }^{30}$ it is the best selection for adefovir 
dipivoxil resistance if the cost can be borne by the patients. However, there are still many problems that need to be studied such as: Can the combination therapy be switched to monotherapy after a fixed time or after the HBV DNA decreases to undetectable level? How about the side effects and how to deal with them after a longer time? How to deal with the viral breakthrough and multidrug resistance after combination therapy?

We will carry out further randomized longer term investigations with larger sample sizes to solve these questions.

\section{Summary}

$\mathrm{LMV}+\mathrm{ADV}, \mathrm{LdT}+\mathrm{ADV}$, and ETV + ADV are all relatively safe and effective rescue strategies for treatment of chronic hepatitis B with adefovir dipivoxil resistance, with $\mathrm{LMV}+\mathrm{ADV}$ as the optimal selection and LdT + ADV as the alternative selection in the economically less developed regions, while ETV + ADV can be used in the economically developed regions.

\section{Acknowledgments}

This work was supported by funding from the National Natural Science Foundation of China (81360080, 30971205, 30672149) and the Major National Basic Research Program of China (2007CB512007).

\section{Disclosure}

The authors report no conflicts of interest in this work.

\section{References}

1. Kuo A, Gish R. Chronic hepatitis B infection. Clin Liver Dis. 2012; 16(2):347-349.

2. Dandri M, Locarnini S. New insight in the pathobiology of hepatitis B virus infection. Gut. 2012;61(Suppl 1):16-17.

3. Arzumanyan A, Reis HM, Feitelson MA. Pathogenic mechanisms in HBV- and HCV-associated hepatocellular carcinoma. Nat Rev Cancer. 2013;13(2):23-35.

4. Ayub A, Ashfaq UA, Haque A. HBV induced HCC: major risk factors from genetic to molecular level. Biomed Res Int. 2013;2013:810461.

5. Zhang QQ, An X, Liu YH, et al. Long-term nucleos(t)ide analogues therapy for adults with chronic hepatitis $\mathrm{B}$ reduces the risk of long-term complications: a meta-analysis. Virol J. 2011;8:72.

6. Thiele M, Gluud LL, Dahl EK, et al. Antiviral therapy for prevention of hepatocellular carcinoma and mortality in chronic hepatitis B, systematic review and meta-analysis. BMJ Open. 2013;3(8):e003265.

7. European Association for the Study of the Liver. EASL Clinical Practice Guidelines: management of chronic hepatitis B. J Hepatol. 2009;50(2):227-242.

8. Wang J. Clinical utility of entecavir for chronic hepatitis B in Chinese patients. Drug Des Devel Ther. 2013;8:13-24.

9. Dienstag JL. Benefits and risks of nucleoside analog therapy for hepatitis B. Hepatology. 2009;49(5 Supp1):S112-S121.

10. Marcellin P, Chang TT, Lim SG, et al. Long-term efficacy and safety of adefovir dipivoxil for the treatment of chronic hepatitis B e antigenpositive chronic hepatitis B. Hepatology. 2008;48(3):750-758.
11. Soriano V, McMahon B. Strategic use of lamivudine in the management of chronic hepatitis B. Antiviral Res. 2013;100(2):435-438.

12. Ghany MG, Doo EC. Antiviral resistance and chronic hepatitis B therapy. Hepatology. 2009;49(5 Suppl):S174-S184.

13. Segovia MC, Chacra W, Gordon SC. Adefovir dipivoxil in chronic hepatitis B: history and current uses. Expert Opin Pharmacother. 2012;13(2):245-254.

14. Yapali S, Talaat N, Lok AS. Management of hepatitis B: our practice and how it relates to the guidelines. Clin Gastroenterol Hepatol. 2014;12(1):16-26.

15. Liu Y, Liu W, Li X, et al. Screening and identification of a novel adefovir dipivoxil resistance associated mutation, rtN236V, of HBV from a large cohort of HBV-infected patients. Antivir Ther. 2014;19(6):551-558.

16. Wang X, Wei F, Hu H, et al. Efficacy and resistance in de novo combination lamivudine and adefovir dipivoxil therapy versus entecavir monotherapy for the treatment-naive patients with chronic hepatitis B: a meta-analysis. Virol J. 2014;11:59.

17. Li W, Huang L, Guo H, Wei X, Liang Z. Antiviral efficacy analysis of telbivudine concurrent with the first cycle of chemotherapy in HBsAgpositive lymphoma patients. J Clin Virol. 2014;61(2):199-203.

18. Osiowy C, Villeneuve JP, Heathcote EJ, et al. Detection of rtN236T and rtA181V/T mutations associated with resistance to adefovir dipivoxil in samples from patients with chronic hepatitis B virus infection by the INNO-LiPA HBV DR line probe assay. J Clin Microbiol. 2006;44(6): 1994-1997.

19. Yu SJ, Kim YJ. Hepatitis B viral load affects prognosis of hepatocellular carcinoma. World J Gastroenterol. 2014;20(34):12039-12044.

20. Lo AO, Wong GL. Current developments in nucleoside/nucleotide analogues for hepatitis B. Expert Rev Gastroenterol Hepatol. 2014;8(6): $607-622$.

21. Lu JJ, Liu K, Ma YJ, et al. Efficacy and safety of telbivudine plus adefovir dipivoxil combination therapy and entecavir monotherapy for $\mathrm{HBeAg-positive} \mathrm{chronic} \mathrm{hepatitis} \mathrm{B} \mathrm{patients} \mathrm{with} \mathrm{resistance} \mathrm{to} \mathrm{adefovir}$ dipivoxil. J Viral Hepat. 2013;20(Suppl 1):40-45.

22. Yim HJ, Hwang SG. Options for the management of antiviral resistance during hepatitis B therapy: reflections on battles over a decade. Clin Mol Hepatol. 2013;19(3):195-209.

23. Kang SH, Yim HJ, Kim HR, et al. Comparison of lamivudine plus adefovir therapy versus entecavir with or without adefovir therapy for adefovir-resistant chronic hepatitis B. J Clin Gastroenterol. 2014; 48(10):889-895.

24. Nguyen NH, Trinh HN, Nguyen TT, et al. Entecavir is safe and effective in patients previously treated with adefovir, including those with adefovir-resistance. J Gastroenterol Hepatol. Epub August 28, 2014.

25. Bang KB, Kim HJ. Management of antiviral drug resistance in chronic hepatitis B. World J Gastroenterol. 2014;20(33):11641-11649.

26. Xu B, Lin L, Xu G, et al. Long-term lamivudine treatment achieves regression of advanced liver fibrosis/cirrhosis in patients with chronic hepatitis B. J Gastroenterol Hepatol. 2015;30(2):372-378.

27. Lee YB, Lee JH, Lee DH, et al. Efficacy of entecavir plus tenofovir combination therapy for chronic hepatitis B patients with multi-drug resistant strains. Antimicrob Pharmacons Chemother. 2014;58(11):6710-6716.

28. Kim YJ, Sinn DH, Gwak GY, et al. Tenofovir rescue therapy for chronic hepatitis B patients after multiple treatment failures. World $J$ Gastroenterol. 2012;18(47):6996-7002.

29. Jiang H, Wang J, Zhao W. Lamivudine versus telbivudine in the treatment of chronic hepatitis B: a systematic review and meta-analysis. Eur J Clin Microbiol Infect Dis. 2013;32(1):11-18.

30. Xu XH, Li GL, Qin Y, et al. Entecavir plus adefovir rescue therapy for chronic hepatitis B patients after multiple treatment failures in real-life practice. Virol J. 2013;10:162. 


\section{Publish your work in this journal}

Drug Design, Development and Therapy is an international, peerreviewed open-access journal that spans the spectrum of drug design and development through to clinical applications. Clinical outcomes, patient safety, and programs for the development and effective, safe, and sustained use of medicines are a feature of the journal, which

has also been accepted for indexing on PubMed Central. The manuscript management system is completely online and includes a very quick and fair peer-review system, which is all easy to use. Visit http://www.dovepress.com/testimonials.php to read real quotes from published authors.

Submit your manuscript here: http://www.dovepress.com/drug-design-development-and-therapy-journal 